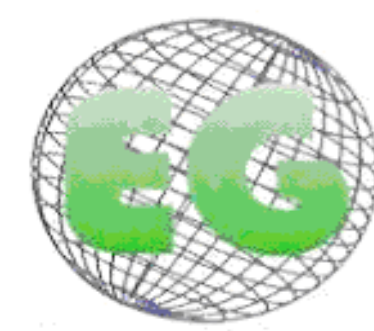

ISSN 1695-6141

$N^{\circ} 23$
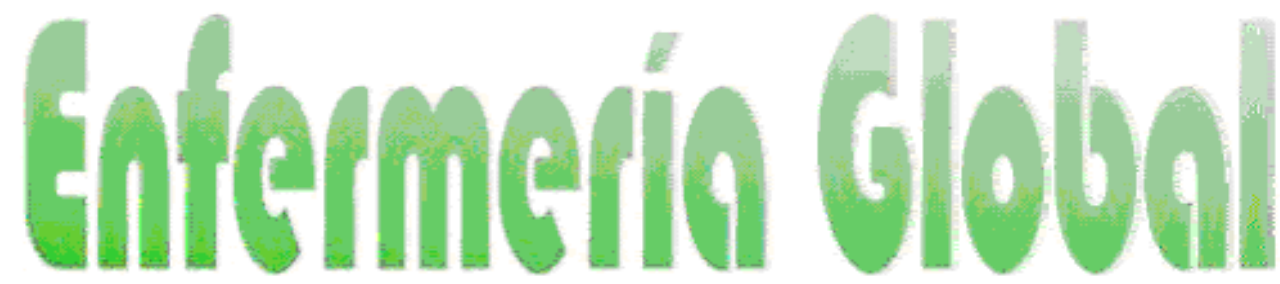

Revista electrónica trimestral de Enfermería

www.um.es/egloball

REVISIONES

\title{
Punción venosa pediátrica. Un análisis crítico a partir de la experiencia del cuidar en enfermería
}

Punção venosa pediátrica: uma análise crítica a partir da experiência do cuidar em enfermagem

\section{*De Oliveira Gomes, AV., **De Luca Nascimento, MA., "* Moreira Christoffel, M., ***Pereira Antunes, JC., ${ }^{* * * *}$ Campos de Araújo, M., ${ }^{* * * *}$ Gomes Cardim, M.}

\author{
*Enfermeira especialista em Enfermagem Pediátrica - IFF/FIOCRUZ. **Doutora em Enfermagem. \\ ${ }^{* * *}$ Mestranda em Enfermagem. **** Mestre em Enfermagem. UNIRIO. Brasil. \\ Palabras clave: Cateterismo periférico; Enfermería pediátrica; Diagnóstico de enfermería; Atención de \\ enfermería.
}

Palavras-chave: Cateterismo Periférico; Enfermagem Pediátrica; Diagnóstico de Enfermagem; Cuidados de Enfermagem

Keywords: Peripheral catheterization; Pediatric Nursing; Nursing Diagnosis; Nursing Care.

\section{RESUMEN}

Estudio descriptivo observacional realizado a partir de la observación de las autoras en sus respectivos ámbitos de trabajo, que tiene por objeto: a) describir los signos observados en los niños sometidos a repetidas punciones venosas periféricas y b) hacer hincapié, a la luz de la literatura consultada, en la importancia de la evaluación clínica de la enfermera para establecer un plan de cuidado para el niño. Desde el cotidiano de la práctica del cuidar se describen, referido a los niños objeto de múltiples punciones venosas periféricas, los signos ubicados en las áreas emocional, física y fisiológica que, según la bibliografía consultada, puede generar sentimientos de miedo y ansiedad, que interfieren en el proceso de crecimiento y desarrollo del niño. Se concluyó que la evaluación clínica de la enfermera es de vital importancia, ya que, a partir de ella, los efectos de las múltiples punciones venosas periféricas en pediatría pueden prevenirse mediante la identificación temprana de los diagnósticos de enfermería y la aplicación de un plan de cuidados adecuados a esta clientela.

\section{RESUMO}

Estudo descritivo observacional realizado a partir da observação das autoras em seus respectivos ambientes de trabalho, que tem como objetivos: a) Descrever os sinais observados na criança submetida às repetidas punções venosas periféricas e; b) Enfatizar, à luz da bibliografia consultada, a importância da avaliação clínica do enfermeiro para estabelecer um plano de cuidados a essa criança. A partir do cotidiano da prática de cuidar foram descritos, em relação às crianças submetidas às múltiplas punções venosas periféricas, sinais alocados nas áreas emocional, física e fisiológica 
que, segundo a bibliografia consultada, podem gerar sentimentos de medo e ansiedade, que interferem no processo do crescimento e desenvolvimento infantil. Conclui-se que, a avaliação clínica do enfermeiro é de vital importância, porque, a partir dela, os efeitos das múltiplas punções venosas periféricas em pediatria podem ser evitadas mediante a identificação precoce do diagnóstico de enfermagem e a implementação de um plano de cuidados adequado a essa clientela.

\section{ABSTRACT}

It is a descriptive observational study made from the authors' observations in their respective works which aims to: a) Describe the signs seen in children submitted to repeated punctures in peripheral venous b) Emphasize, in the light of the literature found, the importance of clinical evaluation of the nurse to establish a plan of care for the child. Starting from the daily practice of caring, the signs located in the emotional, ohysical and psychological areas which according to the literature may generate feelings of fear and anxiety, and which may interfere in the growth process and child development were described in relation to children submitted to multiple punctures in peripheral venous. We concluded that the clinical evaluation of the nurse is very important because, the effects of multiple punctures in peripheral venous of the children can be prevented by early identification of the nursing diagnosis and implementation of a plan of care appropriate to this clientele.

\section{INTRODUCCIÓN}

A partir de la experiencia profesional de las autoras y basadas en estudios científicos se observa que el niño, cuando sometido a un largo período de internación, sufre alteraciones emocionales derivadas de la manipulación excesiva.

Además, el cambio de un ambiente familiar con personas conocidas, a un ambiente diferente con personas desconocidas y donde se realizan procedimientos dolorosos, traumatiza el niño, provocando ansiedad, miedo e inseguridad.

En las últimas décadas, las investigaciones en las áreas de las ciencias médicas, humanas y sociales vienen transformando la asistencia al niño hospitalizado con el desarrollo de nuevas y diferentes perspectivas en relación a su cuidado en el proceso salud-enfermedad, orientando la práctica pediátrica ${ }^{1}$.

En relación a los factores estresantes de la hospitalización, los principales factores incluyen "la separación, la pérdida del control, las lesiones corporales y el dolor": 638.

Corroborando la afirmación arriba referenciada en relación al estrés sufrido por el niño, durante la hospitalización, debido a las frecuentes punciones venosas, se puede afirmar que uno de los procedimientos diagnósticos y terapéuticos que causan dolor es la punción venosa, siendo responsabilidad del enfermero utilizar técnicas adecuadas para reducir el número de esas punciones ${ }^{3}$.

Es importante resaltar que las reacciones al dolor y al sufrimiento físico, son diferentes en cada etapa del desarrollo infantil, siendo importante la observación y la identificación de esos comportamientos por los enfermeros pediatras ${ }^{2}$.

Por otra parte, el acceso venoso periférico presenta como desventaja la dificultad de su manutención cuando es necesaria una terapia prolongada, hecho que exigirá la realización de varias punciones. 
Sin embargo, los niños sometidos a la punción venosa periférica de repetición presentan señales emocionales, físicas y fisiológicas que pueden ser perjudiciales al proceso de crecimiento y desarrollo.

De esta forma, los objetivos de este estudio son: a) Describir las señales observadas en el niño sometido a las repetidas punciones venosas periféricas y; b) Enfatizar, a la luz de la bibliografía consultada, la importancia del análisis clínico del enfermero para establecer un plano de cuidados a ese niño.

\section{REVISIÓN DE LA LITERATURA}

\section{Enfermería y Terapia Intravenosa: conocimientos necesarios para la realización del procedimiento}

Para realizar la terapia intravenosa con eficacia, el enfermero necesita conocer la anatomía y la fisiología de la piel y del sistema venoso, la espesura y la consistencia de la piel de los diversos lugares, saber identificar la respuesta fisiológica del sistema vascular en cuanto a la temperatura y al estrés, además de conocer técnicamente el mecanismo de administración de drogas, y entender la acción de la droga y los efectos adversos para propiciar una administración segura de medicamentos ${ }^{4}$.

Complementando la afirmación anterior, es importante destacar que el enfermero sepa reconocer las complicaciones derivadas de la terapia intravenosa, tales como: embolia, irritación del endotelio vascular, acción del pirogénico, infecciones por contaminantes bacterianos o virales y reacciones anafilácticas ${ }^{5}$.

Además de las precauciones y de la observación de la enfermería con relación al tratamiento IV, los factores que se deben considerar, al prepararse y administrar sustancias en los lactantes y en los niños por la vía IV, incluyen los siguientes: Cantidad de la sustancia a ser administrada; dilución mínima de la sustancia, cuando el niño está en restricción de líquidos; tipo de solución en la cual la sustancia puede ser diluida; intervalo de tiempo en el cual la sustancia se puede administrar con seguridad; velocidad de infusión que el niño y los vasos pueden tolerar con seguridad; capacidad de volumen del equipo; horario en el cual esta u otra sustancia debe ser administrada; compatibilidad de todas las substancias que el niño estuviere recibiendo por vía intravenosa ${ }^{2: 757}$.

Además de eso, durante la terapia intravenosa, complicaciones locales pueden ocurrir, como podemos observar en el siguiente relato: Complicaciones locales en la terapia IV ocurren como reacciones adversas o traumas que se quedan alrededor del sitio de la punción. Las complicaciones locales raramente son graves. Tales complicaciones pueden ser reconocidas precozmente por una evaluación objetiva. Evaluar y monitorear son los componentes clave en intervención precoz. Una buena técnica de punción es el principal cuidado relacionado a la prevención de la mayoría de las complicaciones locales asociadas con la terapia IV 4: 239.

Siendo así, se puede afirmar que, la terapia IV tiene como principal objetivo proporcionar un tratamiento con seguridad y eficacia, considerando las necesidades emocionales y de desarrollo del niño.

\section{Punción Venosa Periférica: conocimientos en pediatría}

En relación a la selección de la vena para realizar la punción, el enfermero debe considerar los siguientes aspectos: facilidad de inserción y acceso, tipo de aguja o catéter que puede 
ser empleado, confort y seguridad del paciente. Además de eso, antes de iniciar la punción es necesario chequear la prescripción médica, lavar las manos, preparar el dispositivo, evaluar al paciente y realizar preparación psicológica, seleccionar el lugar y dilatar la vena. Por último, la punción implica seleccionar la aguja, calzar los guantes, preparar el lugar, perforar la vena, fijar el catéter y realizar la cura. Vale resaltar que las técnicas de punción venosa deben ser realizadas de forma aséptica, con el objetivo de proporcionar una terapia parenteral segura ${ }^{4}$.

Además de eso, para realizar la punción venosa en niños con seguridad, deben ser considerados los siguientes factores antes de seleccionar el local: edad del niño, tamaño del niño, condición de las venas, motivo de la terapia, condiciones generales del paciente, movilidad y nivel de actividad del niño, habilidad motora fina y gruesa del niño, percepción de la imagen corporal, miedo a la mutilación y habilidad cognitiva del niño ${ }^{4}$.

En relación a la selección de la vena para realizar la punción, el enfermero debe considerar los siguientes aspectos: facilidad de inserción y acceso, tipo de aguja o catéter que puede ser empleado, confort y seguridad del paciente.

Las recomendaciones arriba deben ser seguidas por el profesional de salud que realizará tal procedimiento, con la finalidad de evitar o minimizar las posibles complicaciones provenientes de la punción venosa. De esa forma, las posibles complicaciones son: [...] infiltración local, reacciones pirogénicas por la contaminación durante la preparación de la medicación o durante la administración del medicamento; trombosis venosa y flebitis, debido a la acción irritante del medicamento al endotelio o a la formación de coágulos; hematomas, por la extravasación de sangre; necrosis, por la salida de medicamento del vaso, posiblemente por el mal posicionamiento del bisel del dispositivo intravenoso. Tales complicaciones se pueden amenizar mediante una correcta preparación y una infusión lenta ${ }^{6:} 79$.

Es importante resaltar que la punción venosa es un tipo de procedimiento considerado por el niño como una agresión contra él, "pues en la mayoría de las veces es acompañado de dolor o miedo, lo que se traduce en llanto y ansiedad" $6: 77$.

Sin embargo, un ambiente tranquilo y acogedor facilita la aceptación del niño para la realización del procedimiento, además de la importancia en orientar al niño y al acompañante sobre la necesidad del procedimiento, explicando acerca de la indicación, riesgos y beneficios, además de orientar el niño acerca del hecho de que él podrá expresar sus sentimientos y que tendrá que mantenerse inmóvil en tanto que sea posible, con la finalidad de colaborar en la realización del procedimiento ${ }^{6}$.

En el estudio acerca de la elaboración del protocolo, abordando la utilización del juego terapéutico durante la punción venosa, se describieron algunas medidas que pueden ser adoptadas con el niño, con la finalidad de minimizar su sufrimiento, tales como:

1. La preparación del niño debe ser realizada preferiblemente por una enfermera de la unidad, que ya ha establecido previamente una relación de confianza con el niño.

2. Inicie la preparación del niño cerca de 30 minutos antes de la realización del procedimiento.

3. Se presente a los padres del niño y charle con ellos, orientándolos acerca de la necesidad de la punción. 
4. Verifique con los padres cuál es el comportamiento del niño frente a los procedimientos dolorosos ${ }^{7: 79}$.

Por lo tanto, la preparación del niño para la realización de la técnica de punción venosa, o sea, su preparación física y/o emocional es necesaria para minimizar su estrés, además de evitar posibles complicaciones durante la realización del procedimiento.

\section{METODOLOGÍA}

Se trata de un estudio descriptivo observacional, basado en la observación clínica de un procedimiento de la práctica cotidiana del cuidar en Enfermería, procedimiento este, la punción venosa periférica pediátrica. De esa forma, no hubo abordaje de la población blanco, teniendo en vista que, las reflexiones de este estudio resultaron de experiencias de las autoras durante toda la práctica.

En el estudio descriptivo los hechos se observan, registran, analizan, clasifican e interpretan, sin la interferencia del investigador.

Vale resaltar que, el estudio descriptivo observacional de niños sometidos a las repetidas punciones venosas periféricas nos puede traer importantes reflexiones teóricas $y$, a través del análisis de los resultados presentados acerca del cotidiano de la práctica de cuidar, en ambiente hospitalaria, realidades se pueden conocer y modificar.

En la metodología observacional es necesario "conocer el contexto, buscar el sentido de la relación persona-ambiente que en el ocurre e identificar variables que pueden interferir en ese proceso" "9: 304 .

Siendo así, a partir de los efectos adversos descriptos en la literatura y observados en la práctica, se sugieren los cuidados de enfermería que se deben implementar y que son presentados en uno cuadro demostrativo.

\section{Atendiendo a los Objetivos Propuestos}

\section{Señales observados en los niños sometidos a las múltiplas punciones venosas periféricas}

Este estudio tuvo como base la observación de los profesionales en él involucrados, así como los estudios científicos que tratan del tema en foco. Siendo así, atendiendo al primer objetivo propuesto en este estudio, se observó que el niño hospitalizado, sometido a los procedimientos dolorosos, sufre alteraciones emocionales además de otras señales impresas en el cuerpo.

Visto que la punción venosa periférica es un procedimiento rutinario en la unidad hospitalaria, en especial la pediátrica, que tiene por objetivo la administración de medicamentos y colecta de sangre, se vuelve un reto cada vez mayor para los profesionales de salud minimizar el dolor y el sufrimiento de los niños sometidos a tal procedimiento.

Además de eso, el equipo hospitalario no se preocupa en explicar tales procedimientos al niño y, eventualmente, promueven condiciones para minimizar su sufrimiento, disminuyendo los efectos nocivos de la enfermedad y de su tratamiento ${ }^{8}$. 
De esa forma, siendo el enfermero el profesional responsable por la realización de varios procedimientos invasivos, es importante resaltar que él debe dejar de ser sólo un "realizador de cuidados técnicos y pasar a ejercer también su función como facilitador de la experiencia para el niño y para sus padres"8: 398 .

La hospitalización puede generar experiencias traumáticas en el niño debido a una serie de restricciones causadas por el cuadro clínico. Además de eso, están presentes factores adversos e inevitables, tales como: el alejamiento del niño de su vida cotidiana, del ambiente familiar, de los amigos, de la escuela, de los objetos personales, estando sometido a procedimientos dolorosos y desagradables ${ }^{9}$.

Todos los factores anteriormente descritos pueden ser perjudiciales al desarrollo físico y mental del niño, haciendo que haya una regresión en sus habilidades y competencias, lo que puede generar miedo y agresividad.

De esa forma, la experiencia sobrevenida del cotidiano de la práctica de cuidar permitió observar las señales que inciden con mayor frecuencia en los niños sometidos a las repetidas punciones venosas periféricas. Esas señales observadas en la práctica, así como sus causas y los sentimientos generados están expuestos en el cuadro 1. Pero, los referidos sentimientos relacionados a las señales observadas se describieron a partir de la bibliografía consultada.

Cuadro 1 - Cuadro demostrativo de las señales que el niño presenta al someterse a la punción venosa periférica de repetición observadas en el cotidiano de la práctica del enfermero pediatra, sus causas y sus respectivos sentimientos generados.

\begin{tabular}{|c|c|c|}
\hline Señales & Causa & Sentimientos \\
\hline $\begin{array}{l}\text { Llanto al } \\
\text { manoseo }\end{array}$ & \multirow{2}{*}{$\begin{array}{lrr}\text { Trauma causado por } & \text { el } \\
\text { ambiente estresante } & \text { del } \\
\text { hospital y por } & \text { los } \\
\text { procedimientos invasivos y } & \text { y } \\
\text { dolorosos resultando en } & \text { en } \\
\text { dificultad de aproximación de } \\
\text { los profesionales de salud para } \\
\text { la realización } & \text { de } \\
\text { procedimientos, incluso los no } \\
\text { dolorosos. }\end{array}$} & \multirow{8}{*}{ Ansiedad } \\
\hline $\begin{array}{l}\text { Lloriquea } \\
\text { cuando } \\
\text { alguien de } \\
\text { blanco se } \\
\text { acerca }\end{array}$ & & \\
\hline $\begin{array}{l}\text { Frente } \\
\text { fruncida }\end{array}$ & \multirow[t]{3}{*}{$\begin{array}{l}\text { Expresión de miedo y } \\
\text { recusación. }\end{array}$} & \\
\hline $\begin{array}{l}\text { Rostro } \\
\text { contraído }\end{array}$ & & \\
\hline $\begin{array}{c}\text { Agitación } \\
\text { psicomotora }\end{array}$ & & \\
\hline Sudoración & $\begin{array}{l}\text { Las gotas de sudor aparecen } \\
\text { por el cuerpo en señal de } \\
\text { miedo. }\end{array}$ & \\
\hline Hematomas & Extravasación de sangre. & \\
\hline Flebitis & $\begin{array}{l}\text { Acción irritante } \\
\text { medicamento al endotelio o a } \\
\text { la formación de coágulos }\end{array}$ & \\
\hline
\end{tabular}




\begin{tabular}{|c|l|}
\hline $\begin{array}{c}\text { Infiltración } \\
\text { local }\end{array}$ & $\begin{array}{l}\text { Reacciones pirogénicas por la } \\
\text { contaminación durante el } \\
\text { preparo de la medicación o } \\
\text { durante la administración del } \\
\text { medicamento }\end{array}$ \\
\hline $\begin{array}{c}\text { Aumento de } \\
\text { la frecuencia } \\
\text { cardiaca }\end{array}$ & $\begin{array}{l}\text { Son señales provenientes del } \\
\text { miedo y/o del estrés. }\end{array}$ \\
\cline { 1 - 2 } $\begin{array}{c}\text { Aumento de } \\
\text { la frecuencia } \\
\text { respiratoria }\end{array}$ & \\
\hline
\end{tabular}

\section{Miedo y Ansiedad: repercusiones en la vida del niño}

Las innovaciones tecnológicas tienen modificado el perfil de los niños que se internan. Esos niños necesitan de cuidados más complejos y de una evaluación psicosocial con el objetivo de evitar las consecuencias negativas de una hospitalización prolongada ${ }^{2}$.

Además de eso, los sentimientos de miedo y ansiedad, provenientes de lesiones corporales y dolor en los niños, pueden ocasionar consecuencias enormes para la vida adulta, volviéndolas personas más temerosas y con tendencia a evitar cuidados médicos².

Observando el cotidiano de la práctica hospitalaria se puede afirmar que los procedimientos dolorosos producen mucha ansiedad en el niño y esta puede reaccionar a estos procedimientos con intenso desaliento emocional y con resistencia física. Sin embargo, se observa aún que, debido a esta ansiedad, los niños reaccionan de la misma forma a los procedimientos no dolorosos.

Vale resaltar que, "los procedimientos invasivos, dolorosos o no, representan una amenaza de lesión corporal para los preescolares. El temor de mutilación es muy prevalente"”: 644.

Además de eso, se observa el sufrimiento del niño en relación a la manipulación excesiva característico del proceso de hospitalización, muchas veces, a través de su ansiedad ${ }^{10}$.

Siendo así, los factores estresantes de la hospitalización, como frecuentes punciones venosas y manipulación excesiva por diversos procedimientos, pueden ocasionar consecuencias negativas para el niño a corto y largo plazo y "generalmente los niños menores de siete años de edad presentan regresión, ansiedad por la separación, apatía, miedos y disturbios del sueño"2: 646 .

\section{Evaluación Clínica: la identificación del diagnóstico de enfermería y sus posibles intervenciones}

Con relación al segundo objetivo propuesto para el desarrollo del presente estudio, se observa que las señales referidas podrían evitarse a partir de un plan de cuidados de enfermería, corroborando así la importancia de la evaluación clínica del enfermero en el acto de cuidar.

Es importante resaltar que, con la implementación del plan de cuidados, el enfermero posibilitará la preparación del niño para la experiencia de la hospitalización y para los procedimientos, dolorosos o no, provenientes de este proceso. Esos cuidados de enfermería 
deben considerar el hecho de que los niños tienen más miedo a lo desconocido que a lo conocido, haciéndose necesario reducir los elementos desconocidos para minimizar el miedo ${ }^{2}$.

En relación al sentimiento de miedo que la hospitalización provoca en el niño, es importante resaltar que, "cuando los niños no tienen un miedo paralizante con el cual luchar, son capaces de encaminar sus energías para lidiar con otros estreses inevitables de la hospitalización y se benefician así con el potencial de crecimiento de la experiencia"2: 647.

Sabiendo que la hospitalización causa reacciones comportamentales en el niño y que esas reacciones son desencadenadas por los factores estresantes relatados anteriormente, los niños, además de tener características propias, "sufren la influencia del profesional que les asiste durante la internación, y esa influencia puede determinar su conducta de comportamiento" ${ }^{\text {10: }}$.

A partir de la identificación de los diagnósticos de enfermería, se puede establecer precozmente un plan de cuidados al niño sometido a los procedimientos dolorosos frecuentes, en especial la punción venosa periférica, presentándose como una alternativa para minimizar el estrés y la incomodidad en el niño, causado por punciones venosas repetidas.

Este estudio proporcionó la identificación de las señales provenientes de la manipulación excesiva, además de su correlación con sentimientos como el miedo y la ansiedad, que son perjudiciales al proceso de crecimiento y desarrollo del niño.

A partir de los referidos sentimientos, se hizo posible, mediante las experiencias de las autoras en el cotidiano de la práctica de cuidar en enfermería con la clientela pediátrica, asociada a la bibliografía consultada, identificar el diagnóstico de enfermería, así como, elaborar un plan de cuidados, descriptos en el cuadro 2 que, al ser implementado precozmente en la situación específica, reducirá los efectos perjudiciales de los procedimientos y de los eventos estresantes.

\section{Cuadro 2 - CuAdRo DEMOSTRATIVO DEL DIAGNÓSTICO DE ENFERMERÍA IDENTIFICADO POR MEDIO DE LAS SEÑALES OBSERVADAS Y LA ELABORACIÓN DEL PLAN DE CUIDADOS}

\section{Ansiedad/Miedo relacionados a los procedimientos y eventos estresantes, secundarios a las frecuentes punciones venosas periféricas.}

- Preparar el niño emocionalmente antes de la realización de los procedimientos invasivos, de acuerdo con la edad y el nivel de comprensión, con la finalidad de minimizar el miedo del desconocido y promover la cooperación.

- Valorar y facilitar la presencia de los padres durante todo el proceso de la hospitalización.

- Reconocer los miedos de la etapa de desarrollo del niño asociados a los procedimientos para garantizar una intervención apropiada.

- Informar al niño acerca del procedimiento a que va a ser sometido, así como, de todos que él pueda presenciar en el ambiente hospitalario, considerando su edad, con la finalidad de disminuir los miedos y la ansiedad. 
- Utilizar estrategias de enfrentamiento como, por ejemplo, el uso del juego terapéutico.

Los diagnósticos de enfermería pueden ser definidos como conclusiones provenientes de la evaluación en enfermería que establece un perfil del estado de salud del paciente y a partir del cual las intervenciones de enfermería se deben determinar ${ }^{11}$.

Con la finalidad de enfatizar la importancia de la implementación de una rutina hospitalaria que minimice los efectos adversos del procedimiento invasivo, es importante resaltar que "la práctica de la punción venosa pediátrica es aprehendida por el proceso del reproductivismo, y que los libros técnicos se fijan a la esfera del planeamiento de material” ${ }^{\text {"2: }}{ }^{11}$. Ese hecho, en nuestra opinión, puede justificar la ocurrencia de las señales observadas y descritas en este estudio. Además de eso, es importante en nuestra práctica profesional: Discutir el "hacer" en enfermería, atendiendo a las dimensiones científicas contenidas en los procedimientos mecánicos y rutinarios, es ponderar científicamente nuestra profesión, y lo que nos posibilita enseñarla es el aprendizaje que con ella adquirimos ${ }^{12: 12}$.

El enfermero necesita de constante reflexión respecto de su práctica diaria con el objetivo de aumentar su interés científico y eliminar el inconformismo social que representa uno de los factores que impiden la busca de propuestas innovadoras y el crecimiento profesional ${ }^{13}$.

Siendo así, este estudio nos posibilitó afirmar que "la evaluación de la enfermería es el primero paso para identificar los diagnósticos de enfermería y planear los cuidados para cada niño"2: 648.

\section{CONSIDERACIONES FINALES}

Este estudio evidenció, mediante la observación empírica del cotidiano de la práctica de cuidar en enfermería asociada a la literatura especializada, las señales provenientes de frecuentes punciones venosas periféricas que se pueden clasificar como emocionales, físicas y fisiológicas.

Las señales observadas en la práctica pediátrica traen consecuencias graves para el desarrollo psicosocial del niño, al desencadenar sentimientos como el miedo y la ansiedad.

Sin embargo, los factores estresantes del ambiente hospitalario pueden tener sus efectos minimizados cuando el enfermero identifica, precozmente, el diagnóstico de enfermería y establece un plan de cuidados específico para cada niño.

Vale resaltar que la punción venosa en pediatría, a pesar de ser foco de muchos estudios de enfermería, sigue ocupando un lugar preferente en la preocupación de estos profesionales, y que los diagnósticos de enfermería, que son precozmente identificados con la finalidad de establecer un plan de cuidados para minimizar sus efectos, deben ser utilizados a partir de criterios clínicos respaldados en las observaciones de las señales descritas en este estudio.

Siendo así, ese estudio es relevante para el área de la salud del niño, pues sus resultados llevan al profesional de salud, que realiza ese procedimiento, a reflexionar acerca de su práctica, evitando así la ocurrencia de iatrogenias advenidas del reproductivismo. 


\section{REFERENCIAS}

1. Oliveira, BRG de; Collet, N. Criança hospitalizada: percepção das mães sobre o vínculo afetivo criança-família. Rev.latino-am.enfermagem. Ribeirão Preto, v. 7, n. 5, p. 95-102, dezembro 1999.

2. Hockenberry, MJ. Wong, Fundamentos de enfermagem pediátrica. Rio de Janeiro: Elsevier, 2006.

3. Dias, EF; Viana, ACN; Andraus, LMS; Pereira, MS; Barbosa, MA - Utilização do dispositivo intravenoso periférico intermitente em pediatria. Revista Eletrônica de Enfermagem (online), Goiânia, v.2, n.3, jul-dez. 2000. Disponível: http://www.fen.ufg.br/revista

4. Phillips, LD. Manual de terapia intravenosa. $2^{\circ}$ ed. Porto Alegre: Artmed; 2001.

5. Bezerra, LCM; Costa, RP da. Princípios Gerais na Terapêutica Intravenosa em RecémNascidos. In: Terapia Intravenosa em Recém-nascidos: orientações para o cuidado de enfermagem/ [editorial] Silva, G.R.G \& Nogueira, M. F. H. Rio de Janeiro: Cultura Médica: 2004.

6. Collet, N; Oliveira, BRG de. Manual de Enfermagem em Pediatria. Goiânia: AB, 2002.

7. Martins, M do R; Ribeiro, CA; Borba, RIH; Silva, CV da. Protocolo de preparo da criança pré-escolar para punção venosa, com utilização do brinquedo terapêutico. Ver Latino-am Enfermagem, 2001, março; 9 (2): 76-85.

8. Ribeiro, CA; Ângelo, M. O significado da hospitalização para a criança pré-escolar: um modelo teórico. Rev Esc Enferm USP 2005; 39(4):391-400.

9. Carvalho, AM; Fonseca, DG; Begnis, JG; Amaral, AM de. Ludicidade e Saúde - Projeto de Integração Multiprofissional. Anais do $7^{\circ}$ Encontro de Extensão da Universidade Federal de Minas Gerais. Belo Horizonte - 12 a 15 de setembro de 2004.

10. Nascimento, MA de L; Souza, E de F. A criança no hospital: a síndrome da criança com o membro superior imobilizado para infusão venosa. Rio de Janeiro: Atlântica editora, 2007.

11. Bireme. Centro Latino-Americano e do Caribe de Informação em Ciências da Saúde. Biblioteca Virtual em Saúde. DeCS - Descritores em Ciências da Saúde [citado 2006 Ago. 06]. São Paulo: BIREME. Disponível em URL: http://decs.bvs.br.

12. Torres, MM. Punção venosa periférica: avaliação do desempenho dos profissionais de enfermagem de um hospital geral do interior paulista [Dissertação de Mestrado Enfermagem]. Ribeirão Preto (SP): Universidade de São Paulo, 2003.

13. Nascimento, MA de L; Almeida, MFP; Porto, FR; Cardoso, TC dos S. Puncionando a veia bailariana - Revista da Sociedade Brasileira de Enfermagem Pediátrica - v.1, n. -0 - pág 2532, julho/ 2001. 